Commentary/Näätänen: ERPs and attention

\title{
Sensory adaptation and mismatch negativity
}

\section{P. Ullsperger, and T. Baldeweg}

Department of Work Physiology, Central Institute of Occupational Medicine of the GDR, Berlin, DDR 1134, German Democratic Republic

Our commentary will dwell only on the interpretation of the elicitation of mismatch negativity (MMN) because the (automatic) detection of physical features of auditory stimuli represents the crucial precondition for all further steps of processing and thus this factor seems of special importance. In the target article, Näätänen proposes (sect. 3.l.l.) two alternative interpretations for the elicitation of MMN, "refractoriness (sensory adaptation or fatigue)" and "the memory trace explanation." Whereas Näätänen clearly inclines toward the latter, the basic physiological phenomena of sensory adaptation should not be overlooked. From the viewpoint of sensory physiology, adaptation plays an important role, and this should not be confused with refractoriness and fatigue. It is well known that, because of adaptation, the absolute threshold is adjusted to mean stimulus intensity. On the whole, however, adaptation effects an adjustment of the working range of the sensory organ, the working point moves to the steepest part of the "dynamic" characteristic curve with the highest sensitivity to stimulus differences (Keidel 1973).

Helson (1964) stated that adaptation represents a mechanism for acquainting us with changes in the environment. He demonstrated in the framework of his adaptation-level theory that, in addition to the various phenomena of adaptation among sensory systems, there are comparable adaptive phenomena in the central nervous system at all levels of information processing and at the behavioral level, as well.

We propose as an alternative to "the memory trace explanation" that the MMN reflects the distance of a stimulus from the current level of sensory adaptation rather than the distance between memory traces of consecutive stimuli per se. Both standard and deviant stimuli contribute according to their frequency of occurrence to the formation of the current adaptation level.

Accepting this, the results of Sams et al. (1984) are not surprising: A standard stimulus immediately following a deviant stimulus elicits a (small) MMN, suggesting that the occurrence of the deviant stimulus affected the current adaptation level. The 
fact that MMN amplitude is enhanced by a decrease in the deviant stimulus probability (Näätänen et al. 1983) can also be seen as supporting this interpretation. On the other hand, when physically identical stimuli were delivered in unsteady rhythms, no MMN was elicited by additional unpredictably occurring stimuli, that is, the sensory adaptation was not affected (Ullsperger et al. 1985). These changes in rhythm which require a higher order of processing are probably detected by controlled processing mechanisms and reflected by processing negativity and subsequent P300 component.

It is interesting that the amplitude of the P300 component was found to reflect the distance between the attended stimulus information and the current (cognitive) adaptation level (Ullsperger \& Gille 1988), which supports Helson's assumption of similar adaptive phenomena at different levels of information processing. We accordingly suggest that MMN accompanies automatic processing of physical stimulus features and reflects a neuronal process indicating to higher processing levels the deviance of the actual stimulus from the currently adjusted adaptation level. 